\title{
Interior and Exterior Boundaries to the Workspace of Mechanical Manipulators
}

\begin{abstract}
Analytical methods for identifying the boundary to the workspace of serial mechanical manipulators and the boundary to voids in the workspace are presented. The determination of parametric equations of surface patches that envelop the workspace of serial manipulators was presented elsewhere and is extended in this paper to an analytical method for void identification. Because of the ability to identify closed-form surface patches that exist internal and external to the workspace, a mathematical formulation based on the concept of a normal acceleration function is introduced. Admissible motion in the normal direction to a point on a singular surface is delineated and characterized by definiteness properties of a quadratic form. An enclosure bound by surface patches that do not admit normal motion is identified as a void. Several examples are treated using this formulation to illustrate the method.
\end{abstract}

Karim Abdel-Malek

Department of Mechanical Engineering and

Center for Computer-Aided Design

The University of Iowa

Iowa City, IA 52242

amalek@icaen.uiowa.edu

Harn-Jou Yeh

Microtek, Inc.

Taiwan

hjyeh@microtek.com.tw

Saib Othman

National Advanced Driving Simulator

The University of Iowa

Oakdale, IA 52242

othman@nads-sc.uiowa.edu

Keywords: manipulator workspace, voids, singular surfaces, workspace boundary, robotics

\section{INTRODUCTION}

Numerical methods for determining workspace boundaries of serial manipulators have been developed in recent years. Exact computation of the workspace and its boundary is of significant importance because of its impact on manipulator design, manipulator placement in an environment, and manipulator dexterity.

Early investigations of manipulator workspaces were reported by Kumar and Waldron (1981), Tsai and Soni (1981), Gupta and Roth (1982), Sugimoto and Duffy (1982), Spanos and Kohli (1985), Kumar (1985), Gupta (1986), and Davidson and Hunt (1987). The consideration of joint limits in the study of manipulator workspaces was presented in a recent report by Delmas and Bidard (1995). Other works that have dealt with manipulator workspace are reported by Agrawal (1990), Gosselin and Angeles (1990), and Emiris (1993). 
Pennock and Kassner (1993) presented a numerical algorithm for the general study of a planar 3DOF manipulator. More recently, Cecarelli (1995) used an algebraic formulation of a workspace boundary to formulate design equations of three-revolute manipulators and Zhang, et al. (1996) presented the graphical representation of kinematic workspaces.

Recently, Haug et al. (1996) formulated numerical criteria to find the workspace (called the accessible output set) of a general multi-degree-of-freedom system using a continuation method to trace boundary curves suitable for the study of both open- and closed-loop manipulators. The initial criteria for this computational method were presented by Haug et al. (1992) and Wang and Wu (1993).

Although there has been many reports in this field, there are only a few that have addressed the existence of voids in the workspace. The work by Cecarelli and Vinciquerra (1995) using an algebraic formulation to determine the workspace of 4DOF-revolute manipulators exhibits a capability for determining holes and voids in the workspace. Two types of voids were identified, those inside the boundary (called ring voids) and those formed by the rotational motion of the boundary and include the main $\mathbf{z}_{o}$-axis (so-called apple voids).

It is noted here that the importance of the analysis of voids in manipulator workspaces extends to many fields, most importantly to computer-aided design (Abdel-Malek and Yeh 1997), where swept volume techniques can be used to generate solid models. Moreover, identification of voids is necessary in the determination of swept trajectories generated by a manipulator link to avoid collisions. The computation of swept volumes is also important in manufacturing where the $\mathrm{NC}$ verification of machining processes requires the computation of the workspace generated by the tool path on the workpiece.

In recent years, this group has made significant contributions towards the understanding of the workspace of serial manipulators (Abdel-Malek and Yeh 1997; 1997b; 2000, AbdelMalek (1997), Abdel-Malek, et al. 1997; 1999a; 1999b). The main contribution of this formulation is that it yields the exact workspace in closed form. Furthermore, barriers inside the workspace where control difficulties may be encountered are also identified.

In this paper, we further expand the above formulation towards better understanding the identification of internal and external boundaries.

Criteria for determining singular surfaces are established in Section 2. Motion in the normal direction to a singular surface is addressed in Section 3. A quadratic form independent of acceleration vectors is derived in Section 4 and the criteria are stated in Sections 5 and 6. Numerous examples are illustrated.

\section{REVIEW OF SINGULAR SURFACE DETERMINATION}

In order to mathematically address the volume in space generated by the motion of the endeffector, it is necessary to obtain a representation for that motion in terms of joint coordinates (i.e., joint variables). The position vector generated by a point on the end-effector of a $n$ DOF arm is expressed by

$$
\mathbf{G}(\mathbf{q})=\left[\begin{array}{lll}
x(\mathbf{q}) & y(\mathbf{q}) & z(\mathbf{q})
\end{array}\right]^{T}
$$


where $\mathbf{G}(\mathbf{q}) \in \mathbf{R}^{3}$, and $\mathbf{q}=\left[q_{1}, q_{2}, \ldots, q_{n}\right]^{T}$. In fact, $\mathbf{G}(\mathbf{q})$ characterizes the set of points that belongs to the workspace. Singular surfaces/curves comprising the workspace were analytically determined by studying the Jacobian. For the set $\mathbf{G}(\mathbf{q})$, rank deficiency criteria applied to the Jacobian

$$
\mathbf{G}_{\mathbf{q}}=\left[\partial G_{i} / \partial q_{j}\right]
$$

where $G_{i}, i=1,2,3$ are the components of $\mathbf{G}$, yield singular sets $\mathbf{p}^{(i)}$ ( $\mathbf{p}$ have been defined by Abdel-Malek and Yeh 1999) in terms of joint variables. It was shown that substituting $\mathbf{p}^{(i)}$ into $\mathbf{G}(\mathbf{q})$ yields $\mathbf{f}^{(i)}\left(\mathbf{u}^{(i)}\right)$ where $\mathbf{u}^{(i)}$ is the new vector of generalized coordinates such that

$$
\mathbf{f}^{(i)}\left(\mathbf{u}^{(i)}\right)=\mathbf{G}\left(\mathbf{u}^{(i)}, \mathbf{p}^{(i)}\right)
$$

where $\operatorname{dim}(\mathbf{f})=(3 \times 1)$ and $\mathbf{f}^{(i)}$ characterize a closed form equation of a singular surface. It was also shown that these singular curves are used to verify the planned trajectory of a manipulator (Abdel-Malek and Yeh 1997).

A convenient parametrization of constraints imposed on $\mathbf{q}$ was presented such that joint inequality constraints $q_{i}^{L} \leq q_{i} \leq q_{i}^{U}$ are parametrized as

$$
q_{i}\left(\lambda_{i}\right)=\frac{\left(q_{i}^{U}+q_{i}^{L}\right)}{2}+\frac{\left(q_{i}^{U}-q_{i}^{L}\right)}{2} \sin \lambda_{i}
$$

where new parameters were introduced such that $\mathbf{q}=\mathbf{q}(\mathbf{s})$, where $\mathbf{s}=\left[\begin{array}{lll}\lambda_{1} & \lambda_{2} \ldots \lambda_{n}\end{array}\right]^{T}$ and $\mathbf{G}=\mathbf{G}(\mathbf{q}(\mathbf{s}))$ is now a function of the new variables (usually called slack variables). This parametrization is important because it will enable us to include joint limits in the formulation. Note that the limits of a joint are now obtained by varying $\lambda_{i}$ (i.e., $\lambda_{i}=-\pi / 2$ or $\lambda_{i}=\pi / 2$ identifies a limit). An expression for the velocity at any point in the workspace is obtained by deriving $\mathbf{G}(\mathbf{s})$ with respect to time as

$$
\dot{\mathbf{G}}=\mathbf{G}_{\mathbf{q}} \mathbf{q}_{\mathbf{s}} \dot{\mathbf{S}}
$$

where the terminology $\mathbf{q}_{\mathbf{s}}=\partial \mathbf{q} / \partial \mathbf{s}$ and $\dot{\mathbf{s}}=\partial \mathbf{s} / \partial t$. Using the Implicit Function Theorem, the manipulator is at a singular configuration $\mathbf{q}_{o}$ when the rows of $\mathbf{G}_{\mathbf{q}} \mathbf{q}_{\mathbf{s}}$ are dependent and there exists a set of constants $\mathbf{N}_{o}=\left[\begin{array}{lll}\gamma_{1} & \gamma_{2} & \gamma_{3}\end{array}\right]^{T}$ that satisfy

$$
\left[\mathbf{G}_{\mathbf{q}} \mathbf{q}_{\mathbf{s}}\right]^{T} \mathbf{N}_{o}=\mathbf{0}
$$

where $\mathbf{N}_{o}$ is the vector normal to a singular surface at $\mathbf{q}_{o}$.

\section{ADMISSIBLE MOTION}


In this section, the acceleration normal at a point on a singular surface is formulated as a quadratic form. The void identification condition states that a closed boundary for which the acceleration form yields admissible output normals towards the external of the enclosed space for all surfaces enclosing this region, then that space is a void and cannot be reached by the manipulator's end-effector. A point on a singular surface admits motion normal to the surface in either direction depending on the difference in acceleration components (defined by $\eta$ ), such that

$$
\eta=a_{n}-\frac{v_{t}^{2}}{\rho_{o}}
$$

where $v_{t}$ is the tangential velocity, $a_{n}$ is the normal acceleration, and $1 / \rho_{o}$ is the normal curvature of the singular surface (Fig. 1) with respect to the tangent direction of $v_{t}\left(\rho_{o}\right.$ is the radius of curvature). Although it may be unclear at this time, the need for formulating the problem in terms of velocities and accelerations will become apparent. It will be shown that although crossing a barrier is characterized in terms of velocities and accelerations, the crossability criteria is invariant (i.e., not affected by the values of the said quantities).

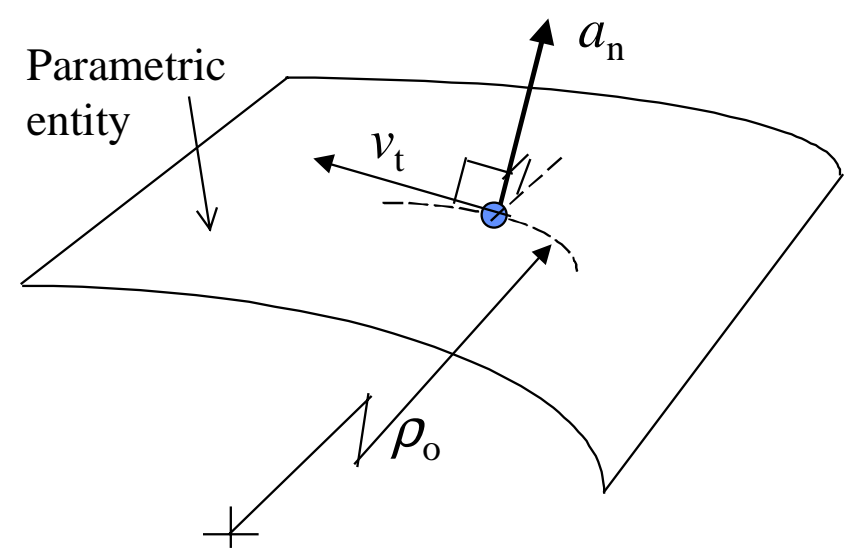

Figure 1 Concept of normal acceleration

A point on a singular surface will have no acceleration if the quantity $\eta$ computes to null. It will be shown that this quantity will indeed identify the boundary to a void since at those singular surfaces, Eq. 7 will indicate no motion into the void, or towards the external of the workspace volume regardless of the value of the velocities or accelerations. Part of a surface enveloping a void in the volume must admit motion only towards the external of that void as shown in Fig. 2. This concept will be generalized in the discussion that follows.

It should be noted that although some barriers may occur in the workspace at which the surface does not admit motion, these barriers do not enclose a region, and therefore, do not form a void. These entities, however, may cause impediments to motion (Haug, et al. 1995) and exhibit control difficulties. 


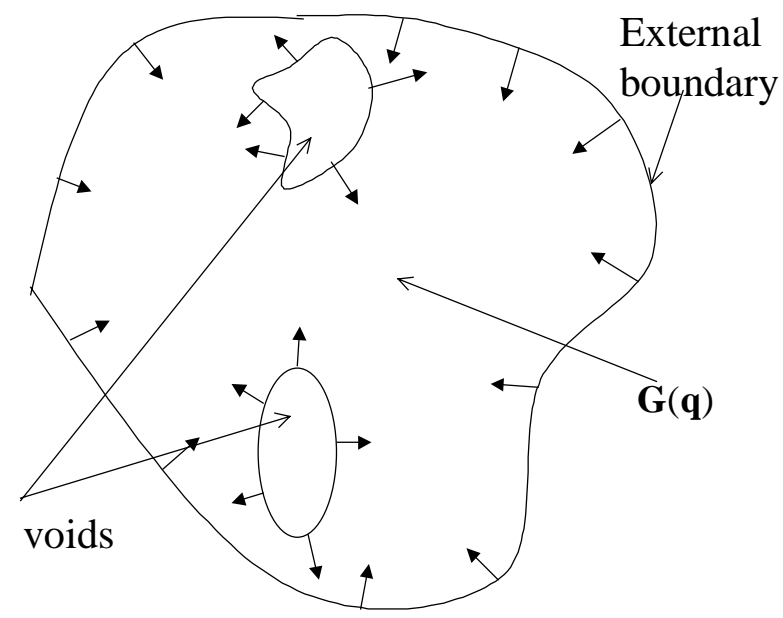

Figure 2 Boundary and void identification

For a singular parametric entity $\mathbf{f}^{(i)}\left(\mathbf{u}^{(i)}\right) \in \mathbf{R}^{3}$ (where $\mathbf{u}$ is indeed a vector representing the remaining joint variables - those not fixed), and in the field of differential geometry, The First Fundamental Form (Farin 1993) is denoted by $\mathbf{I}_{p}$, where $\mathbf{u}=\left[\begin{array}{ll}u & v\end{array}\right]^{T}=\left[\begin{array}{ll}q_{i} & q_{j}\end{array}\right]^{T}$, and is defined as

$$
\mathbf{I}_{p} \equiv \delta \mathbf{u}^{T} \mathbf{f}_{\mathbf{u}}^{T} \mathbf{f}_{\mathbf{u}} \delta \mathbf{u}
$$

where $\mathbf{f}_{\mathbf{u}}=\partial \mathbf{f} / \partial \mathbf{u}$. The Second Fundamental Form is defined as

$$
\mathbf{I I}_{p} \equiv \delta \mathbf{u}^{T}\left[\mathbf{N}^{T} \mathbf{f}\right]_{\mathbf{u u}} \delta \mathbf{u}
$$

or expanded as

$$
\mathbf{I I}_{p}=\mathbf{N}^{T} \mathbf{f}_{u u} d u^{2}+2 \mathbf{N}^{T} \mathbf{f}_{u v} d u d v+\mathbf{N}^{T} \mathbf{f}_{v v} d v^{2}
$$

where $\mathbf{N}$ is the vector normal to the singular surface and $\mathbf{f}_{u v}=\partial^{2} \mathbf{f} / \partial u \partial v$. The Normal Curvature $K_{o}$ of a parametric surface at a configuration $\mathbf{q}_{o}$, in the direction of $d u / d v$, can then be defined as the ratio (Farin 1993)

$$
K_{o}=\frac{1}{\rho_{o}}=\frac{\mathbf{I I}_{p}}{\mathbf{I}_{p}}
$$

In order to define the kinematics quantities, we define the Time-Modified First and Second Fundamental Forms as

$$
\begin{gathered}
\mathbf{I}_{p}^{\prime} \equiv \dot{\mathbf{u}}^{T} \mathbf{f}_{\mathbf{u}}^{T} \mathbf{f}_{\mathbf{u}} \dot{\mathbf{u}} \\
\mathbf{I I}_{p}^{\prime} \equiv \dot{\mathbf{u}}^{T}\left[\mathbf{N}^{T} \mathbf{f}\right]_{\mathbf{u u}} \dot{\mathbf{u}}
\end{gathered}
$$

such that the normal curvature can still be defined as

$$
K_{o}=\frac{1}{\rho_{o}}=\frac{\mathbf{I I}_{p}}{\mathbf{I}_{p}}=\frac{\mathbf{I I}_{p}^{\prime}}{\mathbf{I}_{p}^{\prime}}
$$

For a singular surface $\mathbf{f}$ with its vector of variables $\mathbf{u}$ (i.e., $\mathbf{f}(\mathbf{u})$, the derivative using the chain rule is $\mathbf{f}_{\mathbf{u}} \dot{\mathbf{u}}$. Similarly, for the overall description of the workspace $\mathbf{G}(\mathbf{q})$, the derivative is $\mathbf{G}_{\mathbf{q}} \dot{\mathbf{q}}$. Therefore, at an instant of time, the tangential velocity in terms of $\mathbf{f}$ or $\mathbf{G}$ at any point on a singular surface is 


$$
\mathbf{v}_{t}=\mathbf{f}_{\mathbf{u}} \dot{\mathbf{u}}=\mathbf{G}_{\mathbf{q}} \dot{\mathbf{q}}
$$

where $\dot{\mathbf{u}}=\mathbf{u}_{\mathrm{s}} \dot{\mathbf{s}}$ and $\dot{\mathbf{q}}=\mathbf{q}_{\mathbf{s}} \dot{\mathbf{s}}$. The squared norm of the velocity is

$$
\left|\mathbf{v}_{t}\right|^{2}=\mathbf{v}_{t}^{T} \mathbf{v}_{t}=\dot{\mathbf{u}}^{T} \mathbf{f}_{\mathbf{u}}^{T} \mathbf{f}_{\mathbf{u}} \dot{\mathbf{u}}
$$

which is equal to the Time-Modified First Fundamental Form $\mathbf{I}_{p}^{\prime}$ of Eq. 10. Therefore, $\mathbf{I}_{p}^{\prime}$ can be written as

Substituting for $1 / \rho_{o}$ into $\eta$ yields

$$
\mathbf{I}_{p}^{\prime}=\left|v_{t}\right|^{2}
$$

$$
\eta=a_{n}-\left|v_{t}\right|^{2} \frac{\mathbf{I I}_{p}^{\prime}}{\mathbf{I}_{p}^{\prime}}=a_{n}-\mathbf{I I}_{p}^{\prime}
$$

The objective is to derive an expression for $\eta$ for any singular surface at a specified point. We will first determine an expression for $\mathbf{I I}_{p}^{\prime}$, followed by an expression for $a_{n}$, and finally obtain a quadratic form for $\eta$.

\section{A QUADRATIC FORM FOR THE NORMAL ACCELERATION 4.1 An Expression for the Time-Modified Second Fundamental Form}

Since $a_{n}$ is in terms of $\dot{\mathbf{q}}$ and to express $\mathbf{I I}_{p}^{\prime}$ in terms of $\dot{\mathbf{q}}$, the velocity vector on a singular surface is extracted from Eq. 14. Since $\mathbf{f}_{\mathbf{u}}$ is not square, the velocity is written as

$$
\dot{\mathbf{u}}=\mathbf{B} \mathbf{G}_{\mathbf{q}} \dot{\mathbf{q}}
$$

where $\mathbf{B}$ is the generalized inverse defined by $\mathbf{B}=\left[\mathbf{E f}_{\mathbf{u}}\right]^{-1} \mathbf{E}$ and

$$
\begin{aligned}
& \mathbf{E}=\left[\begin{array}{lll}
1 & 0 & 0 \\
0 & 1 & 0
\end{array}\right] \text { if the first and second rows of } \mathbf{f}_{\mathbf{u}} \text { are independent } \\
& \mathbf{E}=\left[\begin{array}{lll}
1 & 0 & 0 \\
0 & 0 & 1
\end{array}\right] \text { if the first and third rows of } \mathbf{f}_{\mathbf{u}} \text { are independent } \\
& \mathbf{E}=\left[\begin{array}{lll}
0 & 1 & 0 \\
0 & 0 & 1
\end{array}\right] \text { if the second and third rows of } \mathbf{f}_{\mathbf{u}} \text { are independent }
\end{aligned}
$$

Substituting the expression for $\dot{\mathbf{u}}$ into Eq. 12 yields

$$
\begin{aligned}
\mathbf{I I}_{p}^{\prime} & =\dot{\mathbf{u}}^{T}\left[\mathbf{N}^{T} \mathbf{f}\right]_{\mathbf{u u}} \dot{\mathbf{u}} \\
& =\dot{\mathbf{s}}^{T} \mathbf{q}_{\mathbf{s}} \mathbf{G}_{\mathbf{q}} \mathbf{B}^{T}\left[\mathbf{N}^{T} \mathbf{f}\right]_{\mathbf{u u}} \mathbf{B} \mathbf{G}_{\mathbf{q}} \mathbf{q}_{\mathbf{s}} \dot{\mathbf{s}}
\end{aligned}
$$

where $\dot{\mathbf{u}}$ and $\dot{\mathbf{q}}$ were written in terms of $\mathbf{s}$.

\subsection{A Form for $a_{n}$}

Differentiating Eq. 5 again with respect to time yields the acceleration at any point as

$$
\ddot{\mathbf{G}}=\frac{d}{d t}\left(\mathbf{G}_{\mathbf{q}}\right) \mathbf{q}_{\mathbf{s}} \dot{\mathbf{s}}+\mathbf{G}_{\mathbf{q}} \frac{d}{d t}\left(\mathbf{q}_{\mathbf{s}}\right) \dot{\mathbf{s}}+\mathbf{G}_{\mathbf{q}} \mathbf{q}_{\mathbf{s}} \ddot{\mathbf{s}}
$$

or written in expanded form as

$$
\ddot{G}_{i}=\frac{d}{d t}\left[\frac{d G_{i}}{d q_{j}}\right] \frac{d q_{j}}{d s_{k}} \dot{s}_{k}+\left[\frac{d G_{i}}{d q_{j}}\right] \frac{d}{d t}\left(\frac{d q_{j}}{d s_{k}}\right) \dot{s}_{k}+\left[\frac{d G_{i}}{d q_{j}}\right] \frac{d q_{j}}{d s_{k}} \ddot{s}_{k}
$$


Expanding terms in the form of joint rates and collecting similar terms yields

$$
\ddot{G}_{i}=\dot{s}_{n}\left\{\frac{d q_{j}}{d s_{n}}\left[\frac{d^{2} G_{i}}{d q_{m} d q_{j}}\right] \frac{d q_{j}}{d s_{k}}+\frac{d G_{i}}{d q_{j}} \frac{d^{2} q_{j}}{d s_{n} d s_{k}}\right\} \dot{s}_{k}+\left[\frac{d G_{i}}{d q_{j}} \frac{d q_{j}}{d s_{k}}\right] \ddot{s}_{k}
$$

In matrix form, Eq. 22 is written as

$$
\ddot{\mathbf{G}}=\dot{\mathbf{s}}\left\{\mathbf{q}_{\mathbf{s}}^{T} \mathbf{G}_{\mathbf{q q}} \mathbf{q}_{\mathbf{s}}+\sum_{i=1}^{n} \frac{d \mathbf{G}}{d q_{i}} \cdot\left[q_{i}\right]_{\mathrm{ss}}\right\} \dot{\mathbf{s}}+\left[\mathbf{G}_{\mathbf{q}} \mathbf{q}_{\mathbf{s}}\right] \ddot{\mathbf{s}}
$$

To obtain the normal acceleration, $\ddot{\mathbf{G}}$ is projected onto the normal $\mathbf{N}_{o}$. Pre-multiplying both sides of Eq. 23 by $\mathbf{N}_{o}^{T}$ eliminates the last term of the right hand side (definition of the normal as the basis of the null space in Eq. 6). The component of the normal acceleration is

$$
a_{n}=\mathbf{N}_{o}^{T} \ddot{\mathbf{G}}=\dot{\mathbf{s}}^{T} \mathbf{H}^{*} \dot{\mathbf{s}}
$$

where

$$
\mathbf{H}^{*}\left(\mathbf{q}_{o}, \mathbf{s}_{o}\right)=\mathbf{q}_{\mathbf{s}}^{T}\left[\mathbf{N}_{o}^{T} \mathbf{G}\right]_{\mathbf{q q}} \mathbf{q}_{\mathbf{s}}+\sum_{i=1}^{n} \frac{d\left(\mathbf{N}_{o}^{T} \mathbf{G}\right)}{d q_{i}} \cdot\left[q_{i}\right]_{\mathrm{ss}}
$$

\subsection{A Form for $\eta$}

In order to obtain an expression for $\eta$, substitute for $a_{n}$ (from Eq. 24) and for $\mathbf{I I}_{p}^{\prime}$ (from Eq. 20) into Eq. 17, yields

where

$$
\begin{gathered}
\eta=a_{n}-\mathbf{I I}_{\mathbf{p}}^{\prime}=\dot{\mathbf{s}}^{T} \mathbf{Q}^{*} \dot{\mathbf{s}} \\
\mathbf{Q}^{*}=\mathbf{H}^{*}-\mathbf{q}_{\mathbf{s}}^{T} \mathbf{G}_{\mathbf{q}}^{T} \mathbf{B}^{T}\left[\mathbf{N}_{o}^{T} \mathbf{f}\right]_{\mathbf{u u}} \mathbf{B G}_{\mathbf{q}} \mathbf{q}_{\mathbf{s}}
\end{gathered}
$$

where $\mathbf{Q}^{*}$ is a quadratic form. Definiteness properties of the quadratic form of Eq. 27 defines whether motion occurs normal to a singular surface in the direction of $\mathbf{N}^{T}$ at a specified point (i.e., independent of the value of $\dot{\mathbf{s}}$ ). This form will indicate if a singular surface is a boundary to the workspace or boundary to a void. If $\mathbf{Q}^{*}$ is indefinite, the singular surface admits motion along either normal direction and is not a boundary to the workspace.

When $q_{i}$ is at a limit $\left(q_{i}^{L}\right.$ or $q_{i}^{U}$ ), the form characterized by $\mathbf{Q}^{*}$ yields semi-definite forms because $\dot{q}_{i}=\left[q_{i}^{\max }-q_{i}^{\min } / 2\right] \cos \lambda_{i}=0$ at an upper or lower bound since at a joint limit, $\lambda_{i}=-\pi / 2$ or $\lambda_{i}=\pi / 2$. Semi-definite forms are addressed in the following section.

\section{SEMI-DEFINITE QUADRATIC FORM AT $q_{i}=q_{i}^{\text {limit }}$}

In the case of a joint at its limit, Eq. 26 yields a semi-definite quadratic form. For this case, project a variational motion $\delta \mathbf{G}=\mathbf{G}_{q_{i}} \delta q_{i}$ due to $\delta q_{i}$ onto the normal direction $\mathbf{N}_{o}^{T}$ such that the normal component is given by

$$
\sigma=\mathbf{N}_{o}^{T} \mathbf{G}_{q_{i}} \delta q_{i}
$$

where $\delta q_{i}$ is a specified magnitude of \pm 1 as follows

$$
\delta q_{i}= \begin{cases}+1 & \text { if } q_{i} \text { is at lower bound } \\ -1 & \text { if } q_{i} \text { is at upper bound }\end{cases}
$$

Positive values of $\sigma$ in Eq. 29 indicate that the singular surface admits motion in the positive direction of $\mathbf{N}_{o}^{T}$. However, if the normal vector $\mathbf{N}_{o}^{T}$ is perpendicular to $\mathbf{G}_{q_{i}}$ the indicator $\sigma$ 
in Eq. 28 evaluates to zero. In this case, the normal curvature of the singular surface with respect to the tangent direction of $\mathbf{G}_{q_{i}} \delta q_{i}$ is compared with the normal curvature of the parametric curve when only $q_{i}$ is varying (other parameters are held constant).

To determine the normal direction of admissible movement, it is necessary to evaluate the sign of the difference in the normal curvature. Substituting for the first and second fundamental forms (Eqs. 9 and 10) into the normal curvature (Eq. 11) yields

$$
\mathrm{K}_{2}-\mathrm{K}_{1}=\frac{\delta q_{i}\left[\mathbf{N}^{T} \mathbf{G}\right]_{q_{i} q_{i}} \delta q_{i}}{\delta q_{i} \mathbf{G}_{q_{i}} \mathbf{G}_{q_{i}} \delta q_{i}}-\frac{\delta \mathbf{u}^{T}\left[\mathbf{n}^{T} \mathbf{f}\right]_{\mathbf{u u}} \delta \mathbf{u}}{\delta \mathbf{u}^{T} \mathbf{f}_{\mathbf{u}}^{T} \mathbf{f}_{\mathbf{u}} \delta \mathbf{u}}
$$

where $\mathrm{K}_{1}$ is the normal curvature of $\mathbf{f}$ with respect to the tangent direction $\mathbf{G}_{q_{i}} \partial q_{i}$, and $\mathbf{K}_{2}$ is the normal curvature of the parametric curve when $q_{i}$ is varying while other parameters are held constant. Both curves of the right-hand side of Eq. (30) must have the same tangent vector given by Eq. (14) such that

$$
\mathbf{f}_{\mathbf{u}} \delta \mathbf{u}=\mathbf{G}_{q_{i}} \partial q_{i}
$$

where $\delta q_{i}$ is selected as in Eq. 29. Rearranging Eq. 31 and using the generalized inverse defined in Eq. 18, the vector $\delta \mathbf{u}$ can be written as

$$
\delta \mathbf{u}=\mathbf{B G}_{q_{i}} \delta q_{i}
$$

Substituting Eq. (2) into Eq. (30) and simplifying yields

$$
\mathrm{K}_{2}-\mathrm{K}_{1}=\frac{\delta q_{i}\left[\mathbf{N}_{o}^{T} \mathbf{G}\right]_{q_{i} q_{i}} \delta q_{i}-\delta q_{i} \mathbf{G}_{q_{i}}^{T} \mathbf{B}^{T}\left[\mathbf{N}_{o}^{T} \mathbf{G}\right]_{\mathbf{u u}} \mathbf{B} \mathbf{G}_{q_{i}} \delta q_{i}}{\delta q_{i}^{T} \mathbf{G}_{q_{i}}^{T} \mathbf{G}_{q_{i}} \delta q_{i}}
$$

Since the denominator of the right-hand side of Eq. 33 is always positive, the sign is dependent on the numerator. Define the numerator as $\mu$ where

$$
\mu=\delta q_{i}\left[\mathbf{N}_{o}^{T} \mathbf{G}\right]_{q_{i} q_{i}} \delta q_{i}-\delta q_{i} \mathbf{G}_{q_{i}}^{T} \mathbf{B}^{T}\left[\mathbf{N}_{o}^{T} \mathbf{G}\right]_{\mathbf{u u}} \mathbf{B} \mathbf{G}_{q_{i}} \delta q_{i}
$$

and can be written as

$$
\mu=\left\{\left[\mathbf{N}_{o}^{T} \mathbf{G}\right]_{q_{i} q_{i}}-\mathbf{G}_{q_{i}}^{T} \mathbf{B}^{T}\left[\mathbf{N}_{o}^{T} \mathbf{G}\right]_{\mathbf{u u}} \mathbf{B} \mathbf{G}_{q_{i}}\right\} \delta q_{i}^{2}
$$

Since $\delta q_{i}^{2}$ is always positive, the sign of $\mathrm{K}_{1}-\mathrm{K}_{2}$ depends on the term inside the brackets.

Define $\quad \mu=\bar{K} \delta q_{i}^{2}$

where

$$
\bar{K}=\mathbf{G}_{q_{i}}^{T} \mathbf{B}^{T}\left[\mathbf{N}_{o}^{T} \mathbf{G}\right]_{\mathbf{u u}} \mathbf{B} \mathbf{G}_{q_{i}}-\left[\mathbf{N}_{o}^{T} \mathbf{G}\right]_{q_{i} q_{i}}
$$

Therefore, if $\bar{K}>0$, the singular surface can admit motion into the positive direction of $\mathbf{N}_{o}^{T}$. If $\bar{K}<0$, the singular surface admits motion into the negative direction of $\mathbf{N}_{o}^{T}$.

\section{CRITERIA}

\subsection{Crossability Criteria}

(1) If $\mathbf{Q}^{*}$ is indefinite (has both positive and negative eigenvalues), the singular surface admits motion for each parameter $q_{i}$ which is at its limit, and is not a boundary.

(2) If $\mathbf{Q}^{*}$ is either positive semi-definite or negative semi-definite, the following additional criteria must be evaluated. 
(a) If $\mathbf{N}_{o}$ is not perpendicular to $\mathbf{G}_{q_{i}}$, then $\sigma$ in Eq. 30 must be evaluated

(b) If $\mathbf{N}_{o}$ is perpendicular to $\mathbf{G}_{q_{i}}$, then $\bar{K}$ in Eq. 38 must be evaluated.

(i) If any of $\sigma$ or $\bar{K}$ has a different sign than the nonzero eigenvalues of $\mathbf{Q}^{*}$, the singular surface admits motion along its normal.

(ii) If $\sigma$ and $\bar{K}$ have the same sign as the nonzero eigenvalues of $\mathbf{Q}^{*}$, the singular surface does not admit motion along its normal. The direction for admitting motion in the positive or negative direction of $\mathbf{N}_{o}$, is determined by the sign of $\sigma$ and $\bar{K}$.

\subsection{Void Criteria}

An enclosure in the workspace of a spatial manipulator or an area in the workspace of a planar manipulator that is bound by geometric entities that do not admit motion towards this enclosure or region is defined as a void. This also includes the definition of the external boundary as it will also exhibit a connected region or connected surfaces that enclose a volume and that do not admit motion towards the external.

\section{EXAMPLES}

\subsection{A 4R-Manipulator}

Consider the 4R serial manipulator shown in Fig. 3 with joint constraints as follows $-90^{\circ} \leq q_{4} \leq 90^{\circ},-180^{\circ} \leq q_{3} \leq 30^{\circ}, 0 \leq q_{2} \leq 330^{\circ}$, and $0 \leq q_{1} \leq 210^{\circ}$.

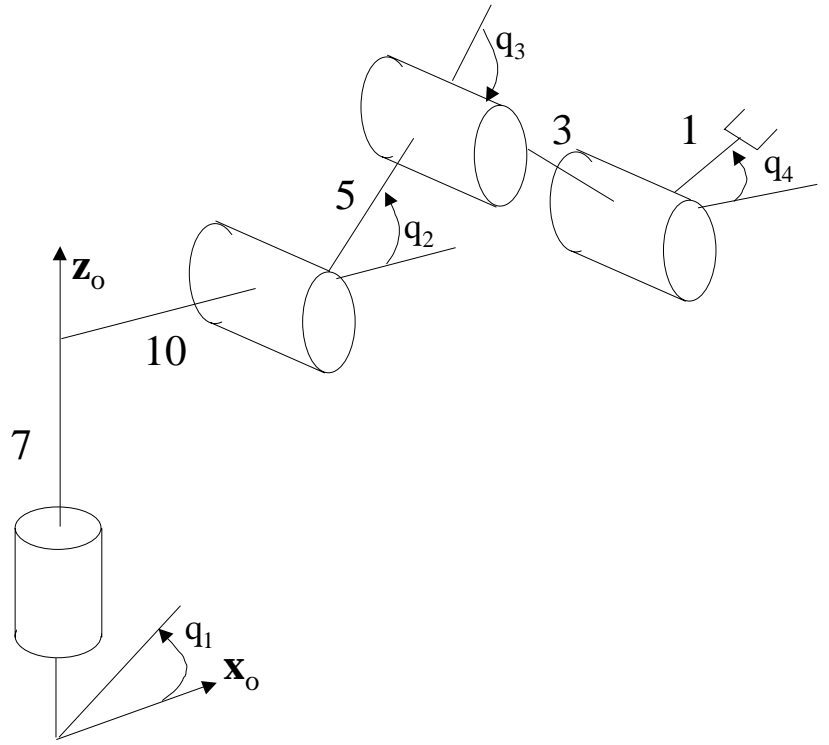

Figure 3 A 4R manipulator

The position vector function characterizing its workspace is given by

$$
\mathbf{G}(\mathbf{q})=\left[\begin{array}{c}
\cos q_{1}\left(10+5 \cos q_{2}+3 \cos \left(q_{2}+q_{3}\right)+\cos \left(q_{2}+q_{3}+q_{4}\right)\right) \\
\sin q_{1}\left(10+5 \cos q_{2}+3 \cos \left(q_{2}+q_{3}\right)+\cos \left(q_{2}+q_{3}+q_{4}\right)\right) \\
7+5 \sin q_{2}+3 \sin \left(q_{2}+q_{3}\right)+\sin \left(q_{2}+q_{3}+q_{4}\right)
\end{array}\right]
$$


Jacobian rank-deficiency conditions resulting from the row rank deficiency of $\left[\mathbf{G}_{\mathbf{q}}\right]^{T}$ presented in Abdel-Malek and Yeh (1997) yield the following singular sets.

$\mathbf{p}^{(1)}=\left\{q_{2}=0, q_{3}=-\pi\right\}, \mathbf{p}^{(2)}=\left\{q_{2}=0, q_{3}=-\pi / 6\right\}, \mathbf{p}^{(3)}=\left\{q_{2}=0, q_{4}=-\pi / 2\right\}, \mathbf{p}^{(4)}=\left\{q_{2}=0, q_{4}=\pi / 2\right\}$,

$\mathbf{p}^{(5)}=\left\{q_{3}=-\pi, q_{4}=-\pi / 2\right\}, \mathbf{p}^{(6)}=\left\{q_{3}=-\pi, q_{4}=\pi / 2\right\}, \mathbf{p}^{(7)}=\left\{q_{3}=\pi / 6, q_{4}=-\pi / 2\right\}, \mathbf{p}^{(8)}=\left\{q_{3}=\pi / 6, q_{4}=\pi / 2\right\}$,

$\mathbf{p}^{(9)}=\left\{q_{2}=33 \pi / 18, q_{3}=-\pi\right\}, \mathbf{p}^{(10)}=\left\{q_{2}=33 \pi / 18, q_{3}=\pi / 6\right\}, \mathbf{p}^{(11)}=\left\{q_{2}=33 \pi / 18, q_{4}=-\pi / 2\right\}$,

$\mathbf{p}^{(12)}=\left\{q_{2}=33 \pi / 18, q_{4}=\pi / 2\right\}, \mathbf{p}^{(13)}=\left\{q_{3}=0.3217, q_{4}=-\pi / 2\right\}, \mathbf{p}^{(14)}=\left\{q_{3}=-0.3217, q_{4}=\pi / 2\right\}$,

$\mathbf{p}^{(15)}=\left\{q_{3}=-\pi, q_{4}=0\right\}, \mathbf{p}^{(16)}=\left\{q_{3}=\pi / 6, q_{4}=-0.3287\right\}, \mathbf{p}^{(17)}=\left\{q_{2}=0, q_{4}=0\right\}, \mathbf{p}^{(18)}=\left\{q_{2}=33 \pi / 18, q_{4}=0\right\}$,

$\mathbf{p}^{(19)}=\left\{q_{3}=0, q_{4}=0\right\}, \mathbf{p}^{(20)}=\left\{q_{1}^{L}, q_{2}^{L}\right\}, \mathbf{p}^{(21)}=\left\{q_{1}^{L}, q_{2}^{U}\right\}, \mathbf{p}^{(22)}=\left\{q_{1}^{L}, q_{3}^{L}\right\}, \mathbf{p}^{(23)}=\left\{q_{1}^{L}, q_{3}^{U}\right\}$,

$\mathbf{p}^{(24)}=\left\{q_{1}^{L}, q_{4}^{L}\right\}, \mathbf{p}^{(25)}=\left\{q_{1}^{L}, q_{2}^{L}\right\}, \mathbf{p}^{(26)}=\left\{q_{1}^{L}, q_{4}^{U}\right\}, \mathbf{p}^{(27)}=\left\{q_{1}^{U}, q_{2}^{L}\right\}, \mathbf{p}^{(28)}=\left\{q_{1}^{U}, q_{2}^{U}\right\}, \mathbf{p}^{(29)}=\left\{q_{1}^{U}, q_{3}^{L}\right\}$, $\mathbf{p}^{(30)}=\left\{q_{1}^{U}, q_{3}^{U}\right\}, \mathbf{p}^{(31)}=\left\{q_{1}^{U}, q_{4}^{L}\right\}$, and $\mathbf{p}^{(32)}=\left\{q_{1}^{U}, q_{4}^{U}\right\}$. Substituting these singular sets into Eq. 38 yields parametric equations of singular surfaces shown in Fig. 4, and its cross section (at $q_{1}=0$ or $x z$-plane) in Fig. 5.

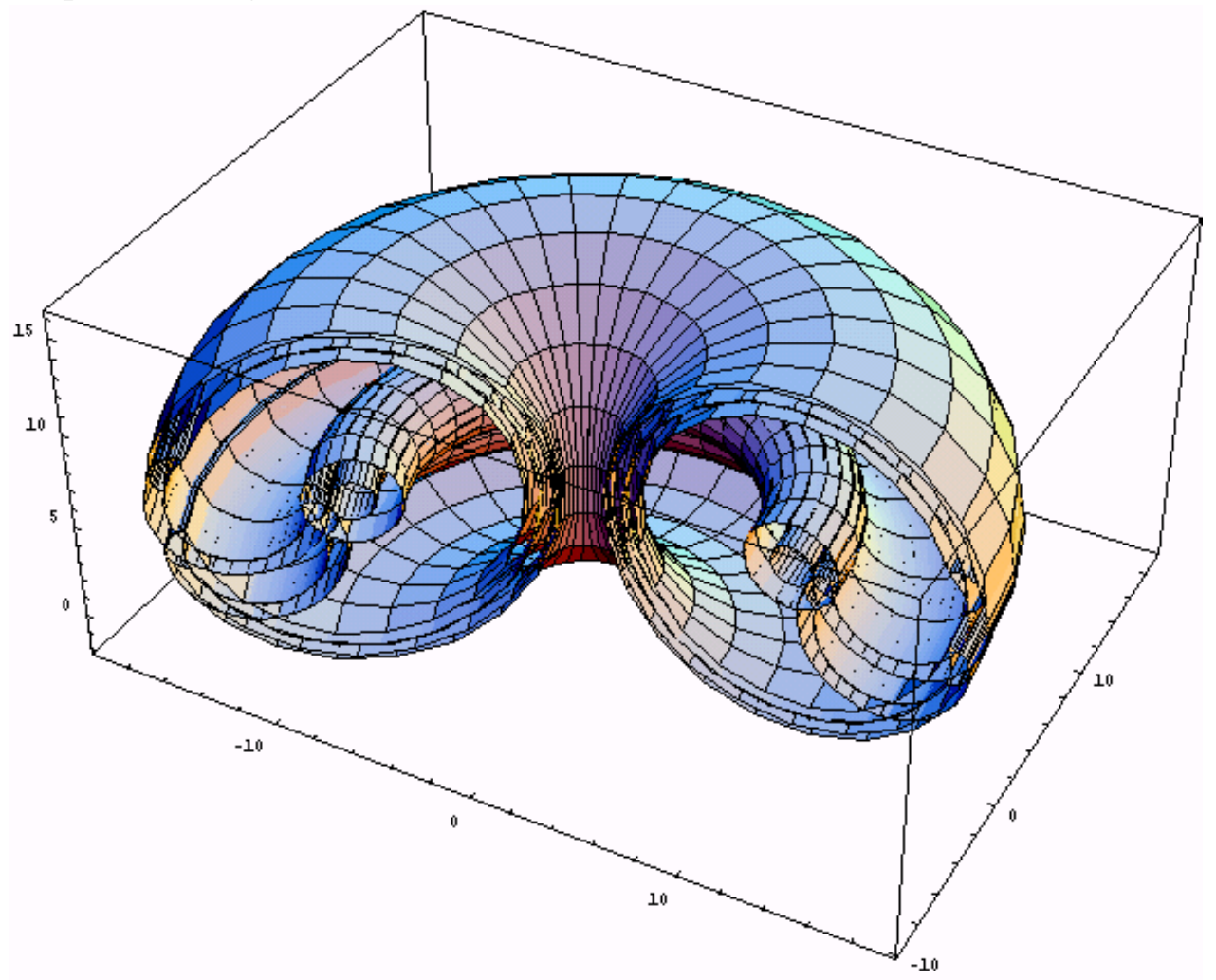

Figure 4 The workspace depicting all singular surfaces 


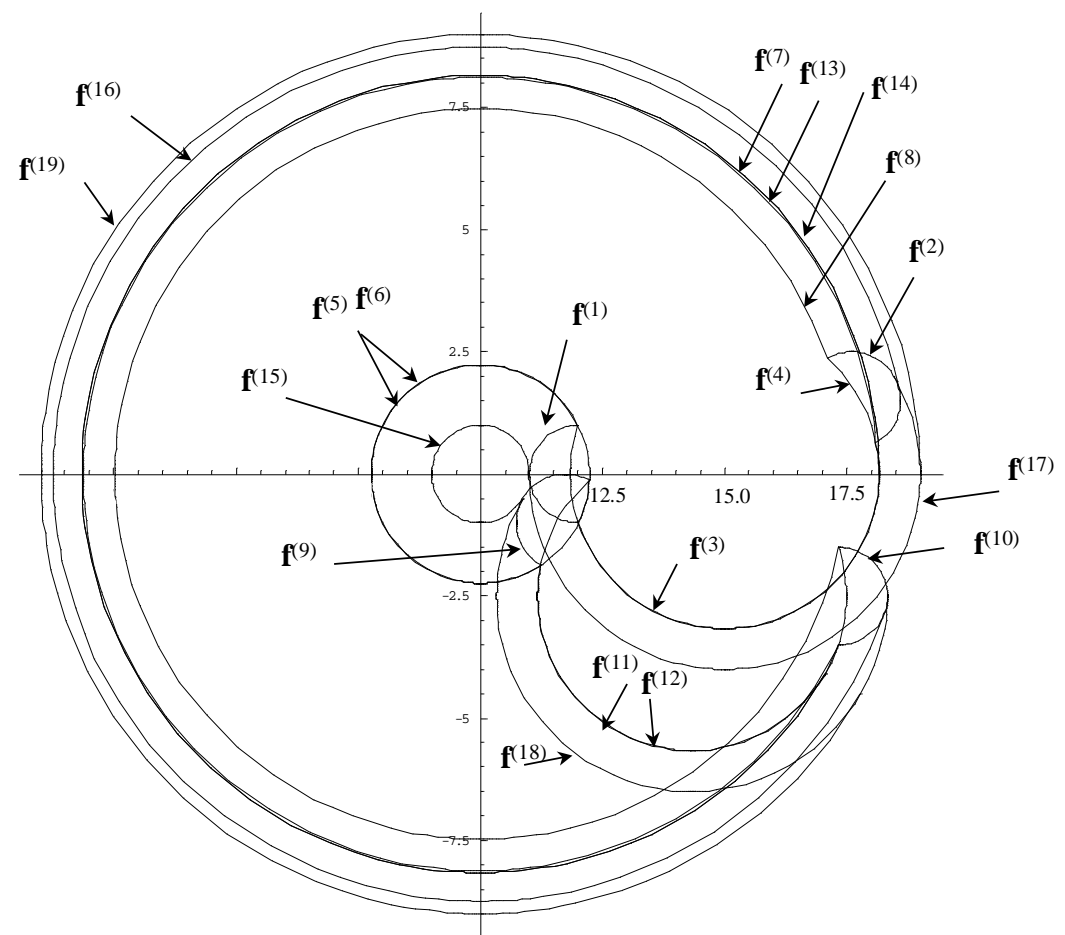

Figure 5 A cross section of the workspace of the 4R manipulator

To determine the boundary to any voids, it is necessary to evaluate the criteria above for all surface patches. Consider for example point $A_{5}$ shown in Fig. 6 on surface $\mathbf{f}^{(15)}$, for which the singular set is $\mathbf{p}^{(15)}=\left\{q_{3}=-\pi, q_{4}=0\right\}$, and the point is evaluated at

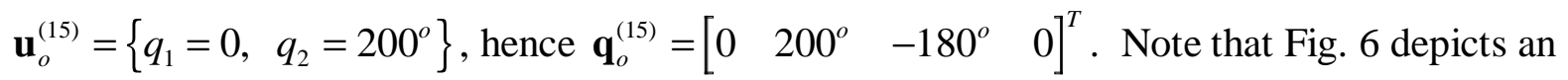
enlarged section of Fig. 5. 


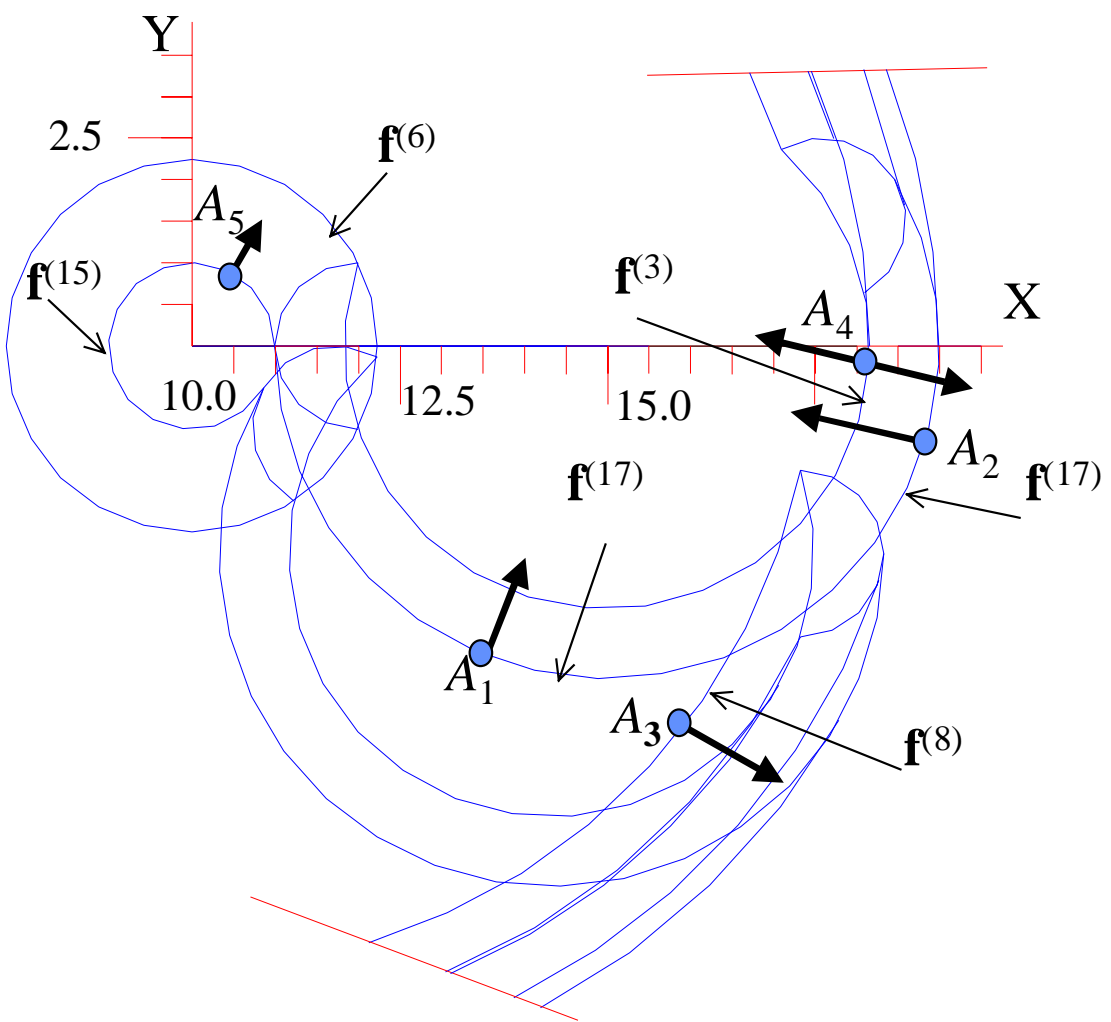

Figure 6 Part of the workspace showing curve segments

Substituting $\mathbf{p}^{(15)}$ into Eq. (38) yield

$\mathbf{f}^{(15)}\left(\mathbf{u}^{(15)}\right)=\left[\begin{array}{lll}10 \cos q_{1}+\cos q_{1} \cos q_{2} & 10 \sin q_{1}+\cos q_{2} \sin q_{1} & 7+\sin q_{2}\end{array}\right]^{T}$, where $\mathbf{u}^{(15)}=\left[\begin{array}{ll}q_{1} & q_{2}\end{array}\right]^{T}$. To compute the normal at $A_{5}$, the matrix $\left[\mathbf{G}_{\mathbf{q}}\left(\mathbf{q}_{o}^{(15)}\right) \mathbf{q}_{\mathbf{s}}\left(\mathbf{s}_{o}^{(15)}\right)\right]^{T}=\left[\begin{array}{ccc}0 & 0 & 0 \\ 0.962 & 0 & -2.644 \\ 0 & 0 & 0 \\ -.0537 & 0 & 1.476\end{array}\right]$ is

computed, and its nullspace is readily determined as $\left\{\begin{array}{llll}0.939 & 0 & 0.342\end{array}\right\},\left\{\begin{array}{lll}0 & 1 & 0\end{array}\right\}$. Since the second vector is trivial, the normal is indeed $\mathbf{n}_{o}^{T}=\left[\begin{array}{lll}0.939 & 0 & 0.342\end{array}\right]$. The matrix $\mathbf{H}^{*}$ is calculated as $\mathbf{H}^{*}=\left[\begin{array}{cccc}0 & 0 & 0 & 0 \\ 0 & 7.920 & 0 & -4.421 \\ 0 & 0 & 0 & 0 \\ 0 & -4.421 & 0 & -2.467\end{array}\right]$ and the generalized inverse is calculated as $\mathbf{B}=\left[\mathbf{E}_{1} \mathbf{f}_{\mathbf{u}}\left(\mathbf{u}_{o}^{(15)}\right)\right]^{-1} \cdot \mathbf{E}_{1}=\left[\begin{array}{ccc}0 & 0.110 & 0 \\ 2.924 & 0 & 0\end{array}\right]$. The final $(4 \times 4) \quad \mathbf{Q}^{*}$ matrix is evaluated and its eigenvalues are computed as $\{-4.935,0,0,0\}$. Since this is a negative semi-definite form, the algorithm must compute a value for $\sigma$. Since $q_{3}=-\pi$ is a lower limit, $\delta q_{3}=+1$ and $\sigma=\mathbf{N}_{o}^{T} \mathbf{G}_{q_{3}}(+1)=0$. Since the normal is along the same direction as the derivative, a value 
for $\bar{K}$ must be computed. The terms are evaluated as follows: $\left[\mathbf{N}_{o}^{T} \mathbf{G}\right]_{q_{3} q_{3}}=-4.0$, $\mathbf{G}_{q_{3}}^{T} \mathbf{B}^{T}=\left[\begin{array}{ll}0 & -4\end{array}\right]$, and $\left[\mathbf{N}_{o}^{T} \mathbf{G}\right]_{\mathbf{u u}} \mathbf{B G}_{q_{3}}=\left[\begin{array}{ll}0 & -4\end{array}\right]^{T}$. Therefore, $\bar{K}=-20.0056$. A negative sign indicates that the singular surface admits movement into the negative direction of $\mathbf{N}_{o}^{T}$. The reader is reminded that the curves shown in Fig. 6 are traces of the surfaces on a plane cutting through the workspace. Those points are indeed positions of the end-effector on a singular surface.

Similarly, for the same singular surface at point $A_{1}$ characterized by $q_{1}=0, q_{3}=-10^{\circ}$ (which is an external boundary), the normal is $\mathbf{N}_{o}^{T}=\left[\begin{array}{llll}-0.985 & 0 & 0.17\end{array}\right]^{T}$ and the eigenvalues are computed as $\{2.5,1.8505,0,0\}$ and $\sigma=0.868$; same sign as the eigenvalues. This indicates an admissible motion in that direction (but not the opposite direction).

On singular surface $\mathbf{f}^{(8)}$ at point $A_{3}$ where $\mathbf{u}^{(8)}=\left\{q_{1}, q_{2}\right\}$, and at $\mathbf{p}^{(8)}=\left\{q_{3}^{U}, q_{4}^{U}\right\}$ and $q_{1}=0, q_{2}=305^{\circ}$, the normal is $\mathbf{N}_{o}^{T}=\left[\begin{array}{lll}-0.8032 & 0 & 0.5957\end{array}\right]^{T}$ and the eigenvalues are computed as $\{-2.8974,-1.5389,0,0\}$ and $\sigma=-1.5810$. On singular surface $\mathbf{f}^{(3)}$ at point $A_{4}$ with $\mathbf{u}^{(3)}=\left\{q_{1}, q_{3}\right\}$, at $\mathbf{p}^{(4)}=\left\{q_{2}^{L}, q_{4}^{U}\right\}$ at $q_{1}=0, q_{3}=0^{\circ}$, and $q_{4}=q_{4}^{L}$, the normal is $\mathbf{N}_{o}^{T}=\left[\begin{array}{lll}-0.9487 & 0 & 0.3160\end{array}\right]^{T}$ and the eigenvalues are computed as $\{4.5529,-1.4901,0,0\}$ which is an indefinite quadratic form. Surface patches that do not admit motion and that enclose a volume, are boundary to the workspace. For this example, there are three sets of boundary surface patches characterized by the following surface regions and illustrated in the cross section of Fig. 7. The workspace with interior and exterior boundaries is shown in Fig. 8.

\begin{tabular}{|l|l|}
\hline The external boundary is characterized by & The boundary to Void 1 \\
$\mathbf{f}^{(19)}\left(q_{1}, q_{2}\right) ; 0 \leq q_{1} \leq 210^{\circ}$ and $0 \leq q_{2} \leq 330^{\circ} ;$ & $\mathbf{f}^{(15)}\left(q_{1}, q_{2}\right) ; 0 \leq q_{1} \leq 210^{\circ}$ and $0^{\circ} \leq q_{2} \leq 330^{\circ}$ \\
$\mathbf{f}^{(17 c)}\left(q_{1}, q_{3}\right) ; 0 \leq q_{1} \leq 210^{\circ}$ and $34^{\circ} \leq q_{3} \leq 7^{\circ}$ & $\mathbf{f}^{(9)}\left(q_{1}, q_{4}\right) ; 0 \leq q_{1} \leq 210^{\circ}$ and $-17^{\circ} \leq q_{4} \leq 0^{\circ}$ \\
$\mathbf{f}^{(10)}\left(q_{1}, q_{4}\right) ; 0 \leq q_{1} \leq 210^{\circ}$ and $-20^{\circ} \leq q_{4} \leq 20^{\circ}$ & $\mathbf{f}^{(17 b)}\left(q_{1}, q_{3}\right) ; 0 \leq q_{1} \leq 210^{\circ}$ and $-\pi \leq q_{3} \leq-175^{\circ}$ \\
$\mathbf{f}^{(18)}\left(q_{1}, q_{3}\right) ; 0 \leq q_{1} \leq 210^{\circ}$ and $0^{\circ} \leq q_{3} \leq 30^{\circ}$ &
\end{tabular}

The boundary to the void is characterized by the following surface patches:

$\mathbf{f}^{(5)}\left(q_{1}, q_{2}\right) ; 0 \leq q_{1} \leq 210^{\circ}$ and $276^{\circ} \leq q_{2} \leq 283^{\circ}$,

$\mathbf{f}^{(17 a)}\left(q_{1}, q_{3}\right) ; 0 \leq q_{1} \leq 210^{\circ}$ and $-154^{\circ} \leq q_{3} \leq-70^{\circ}$,

$\mathbf{f}^{(8)}\left(q_{1}, q_{2}\right) ; 0 \leq q_{1} \leq 210^{\circ}$ and $293^{\circ} \leq q_{2} \leq 312^{\circ}$, and

$\mathbf{f}^{(11)}\left(q_{1}, q_{3}\right) ; 0 \leq q_{1} \leq 210^{\circ}$ and $-142^{\circ} \leq q_{3} \leq-30^{\circ}$. 


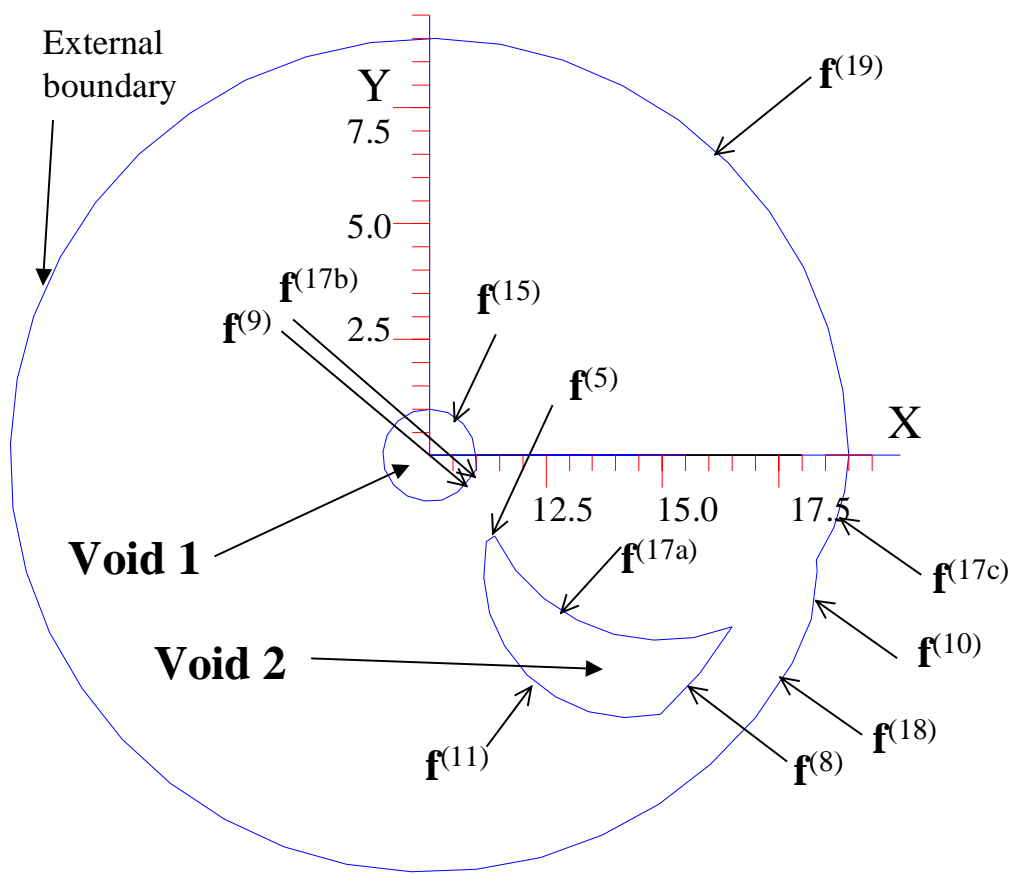

Figure 7 A cross section of the workspace depicting the boundary

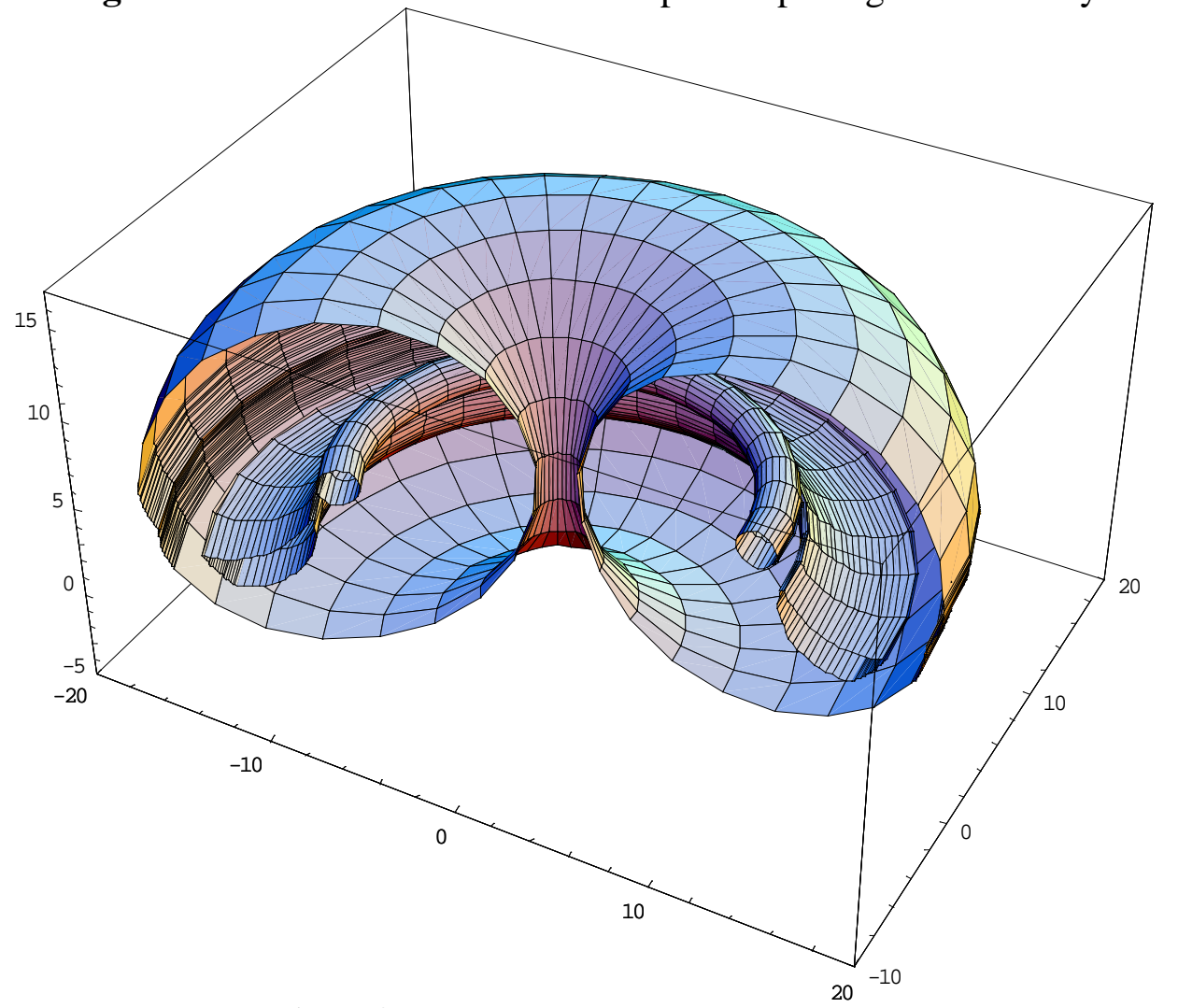

Figure 8 The workspace of the $4 \mathrm{R}$ manipulator 


\subsection{Identifying the boundary of a general 4-DOF}

Consider the general serial 4-DOF manipulator shown in Fig. 9 that was presented by Ceccarelli and Vinciguerra (1995). This example is treated here for validation purposes and to demonstrate the ability of the proposed method in determining the external boundary.

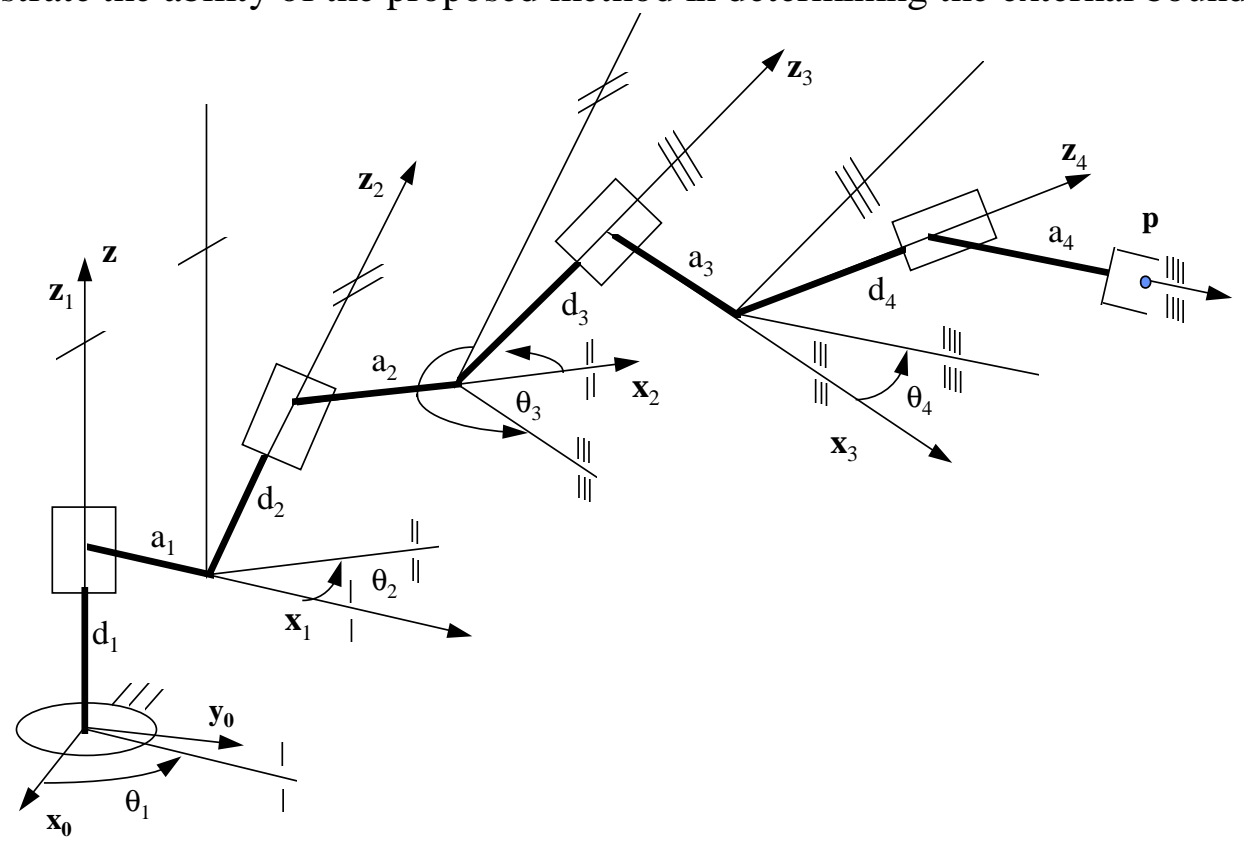

Figure 9 The general 4-DOF serial manipulator

Applying the rank deficiency criteria to $\mathbf{G}_{\mathbf{q}}(\mathbf{q})$ yields singular sets when substituted into $\mathbf{G}(\mathbf{q})$ result in singular surfaces (examples are shown in Fig. 10). The trace of these surfaces on an $x y$-plane at $z=1.0$ is shown in Fig. 11a and the exterior boundary is shown in Fig. 11b.

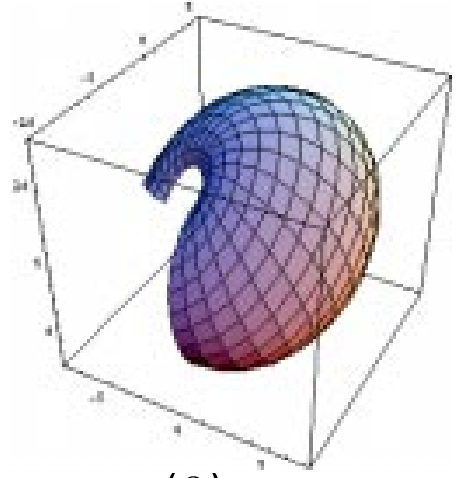

(a)

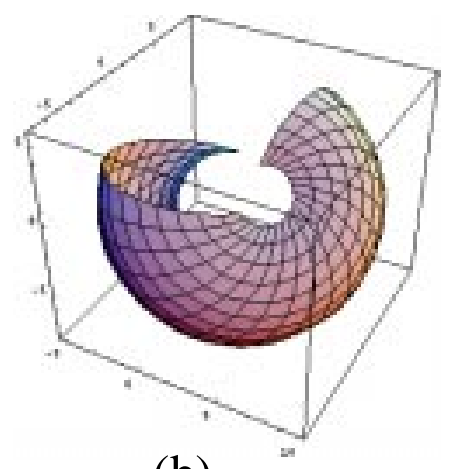

(b)

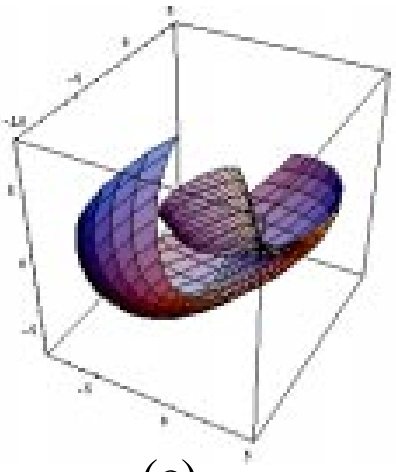

(c)

Figure 10 Singular surface 

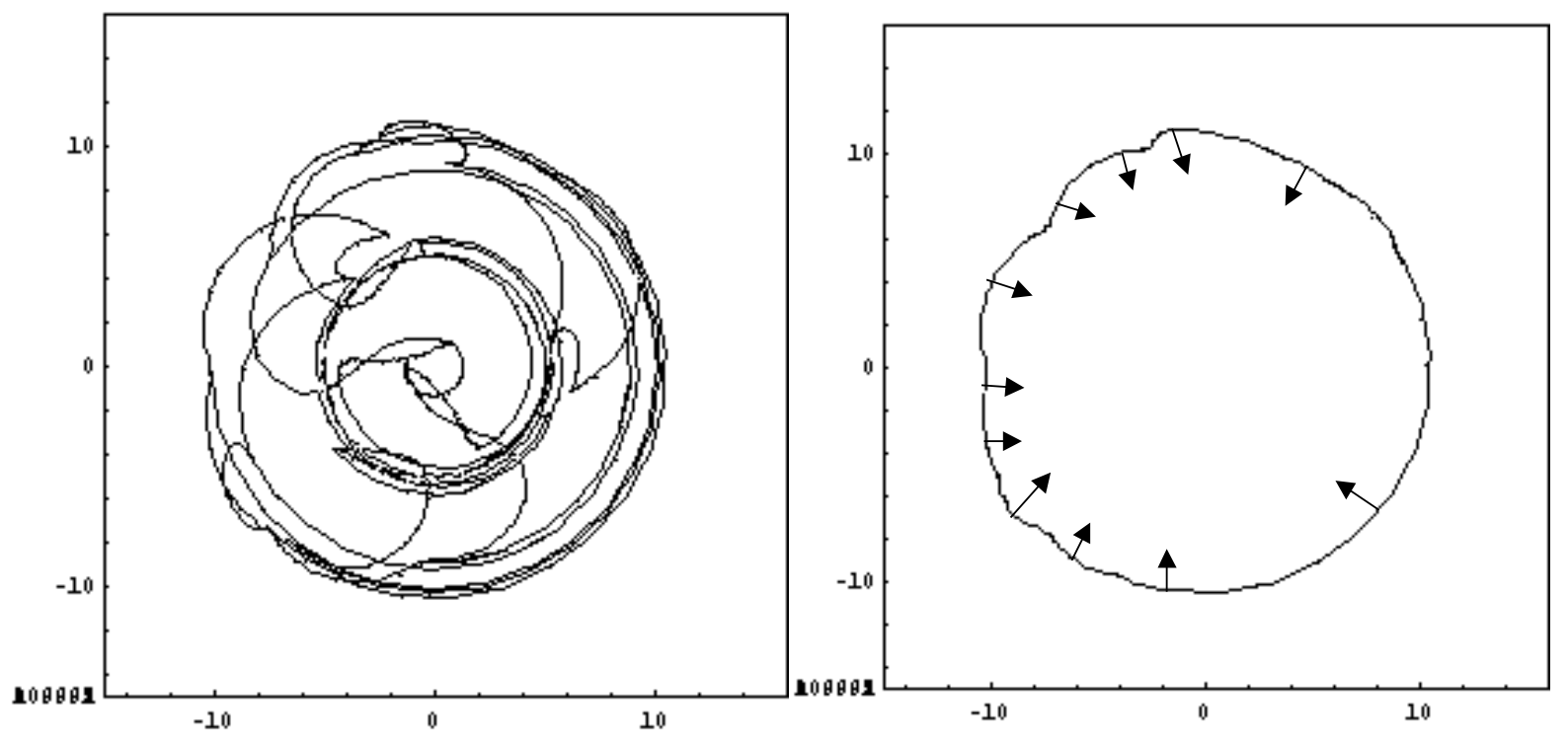

Figure 11 (a) A cross section of the general 4-DOF (b) Identification of the boundary

\subsection{A 5-DOF RPPRR Manipulator}

Consider the 5-DOF manipulator shown in Fig. 12.

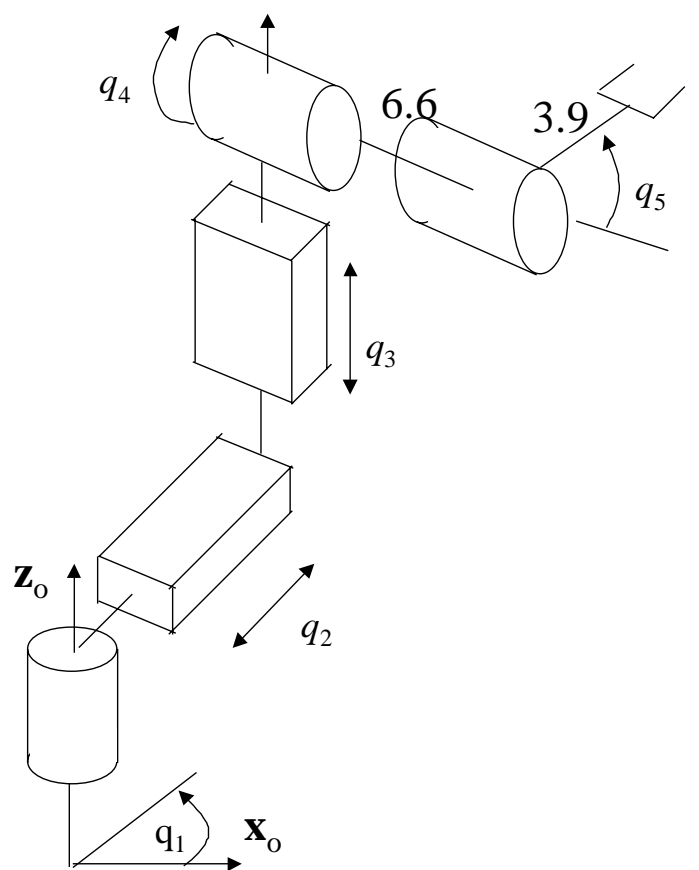

Figure 12 A five-DOF manipulator

The vector function of the position of the end-effector is written as

$\mathbf{G}(\mathbf{q})=\left[\begin{array}{c}-\left(13+q_{2}\right) \sin q_{1}-6.6 \cos q_{4} \sin q_{1}-3.9 \cos q_{4} \cos q_{5} \sin q_{1}+3.9 \sin q_{1} \sin q_{4} \sin q_{5} \\ \left(13+q_{2}\right) \cos q_{1}+6.6 \cos q_{4} \cos q_{1}+3.9 \cos q_{4} \cos q_{5} \cos q_{1}-3.9 \cos q_{1} \sin q_{4} \sin q_{5} \\ 10+q_{3}+6.6 \sin q_{4}+3.9 \cos q_{5} \sin q_{4}+3.9 \cos q_{4} \sin q_{5}\end{array}\right]$ 
and is subjected to the following onstraints: $0 \leq q_{1} \leq 240^{\circ}, 0 \leq q_{2} \leq 2.8,0 \leq q_{3} \leq 4.5$, $0 \leq q_{4} \leq 330^{\circ}$, and $-153^{\circ} \leq q_{5} \leq 128^{\circ}$. Using the rank deficiency conditions, singular sets are computed and substituted into $\mathbf{G}(\mathbf{q})$. The trace of these surfaces on a $x z$-cutting plane is shown in Fig. 13.

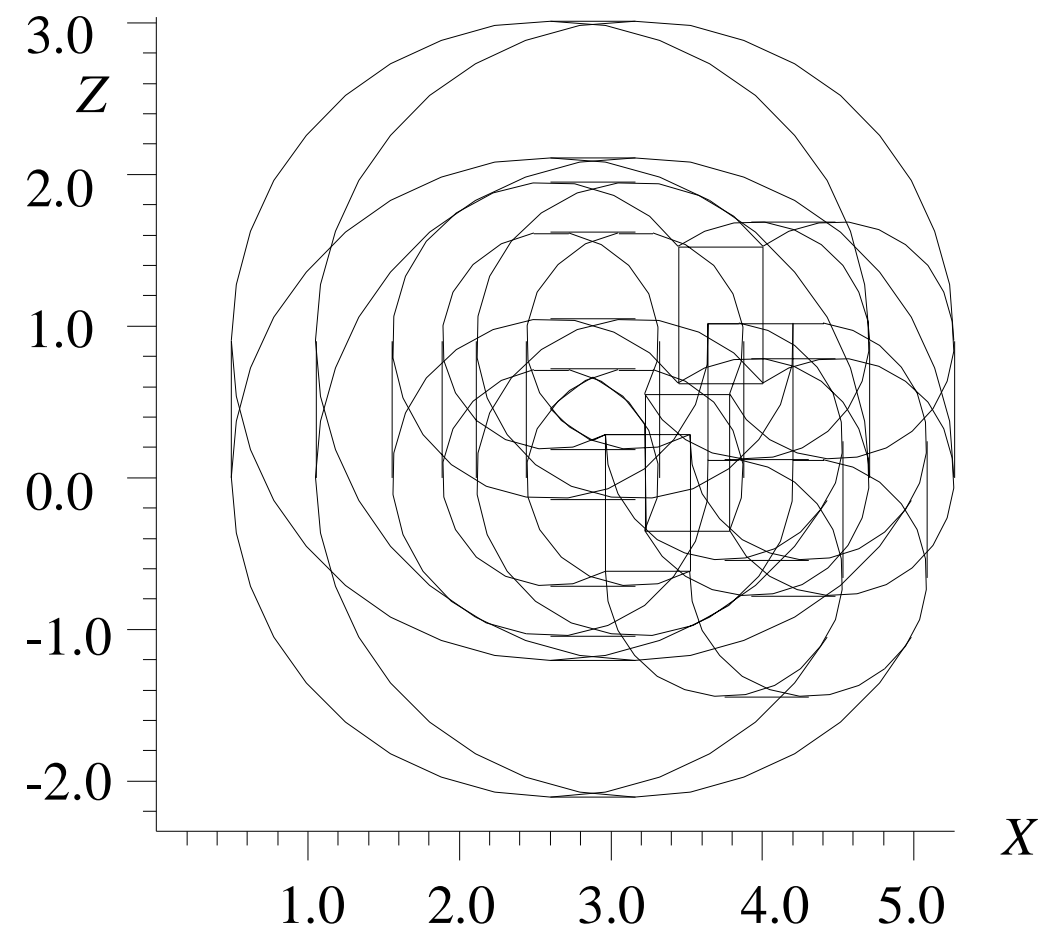

Figure 13 A cross section of the workspace

The normal acceleration analyses applied to each surface patch identifies admissible directions of output motion. Boundary surface patches are identified. Figure shows these patches that are boundary and their admissible normal directions. A cross section of the workspace depicting the external boundary and the void is shown in Fig. 14. 


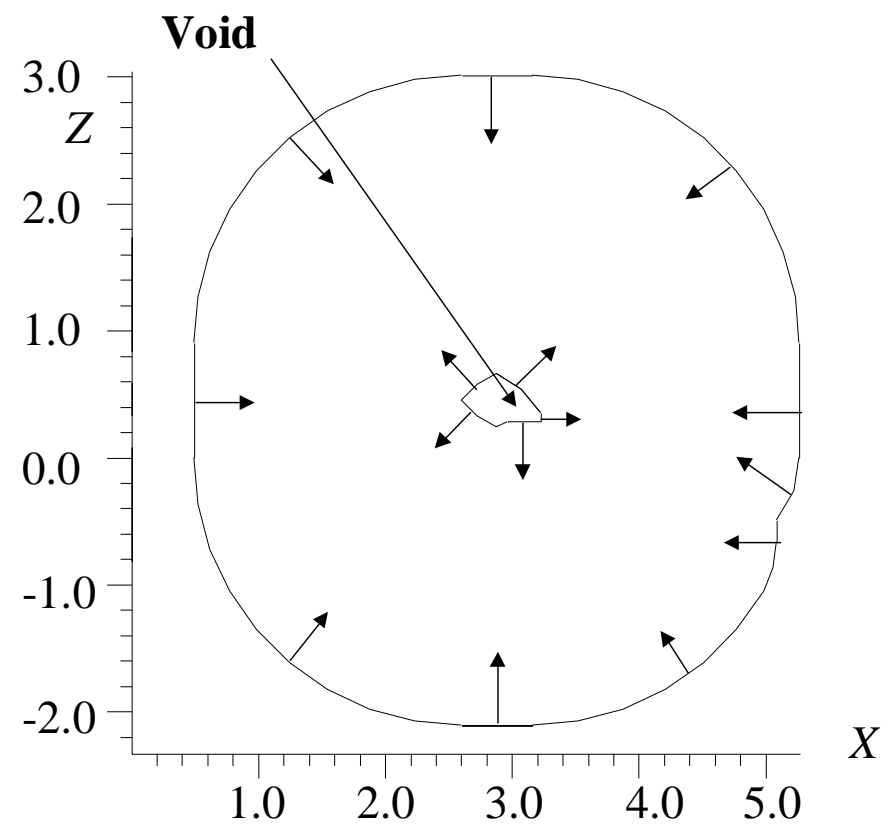

Figure 14 Identifying the external boundary and the void in the workspace

Because the formulation yields parametric surface patches and because the analysis yields a quadratic form that delineates boundary surface patches, it is possible to represent the boundary to the void in an exact form (Figure 15). The boundary surface patches to the void are identified by the following and are depicted in Figure 16.

$$
\begin{aligned}
& \mathbf{f}^{(12)}\left(q_{1}, q_{4}\right), 322^{\circ} \leq q_{4} \leq 330^{\circ} \text { and } 0 \leq q_{1} \leq 240^{\circ} \\
& \mathbf{f}^{(13)}\left(q_{1}, q_{4}\right), 248^{\circ} \leq q_{4} \leq 277^{\circ} \text { and } 0 \leq q_{1} \leq 240^{\circ} \\
& \mathbf{f}^{(14)}\left(q_{1}, q_{4}\right), 142^{\circ} \leq q_{4} \leq 171^{\circ} \text { and } 0 \leq q_{1} \leq 240^{\circ} \\
& \mathbf{f}^{(15)}\left(q_{1}, q_{2}\right), 0 \leq q_{2} \leq 1.3 \text { and } 0 \leq q_{1} \leq 240^{\circ} \\
& \mathbf{f}^{(16)}\left(q_{1}, q_{4}\right), 59^{\circ} \leq q_{4} \leq 97^{\circ} \text { and } 0 \leq q_{1} \leq 240^{\circ} \\
& \mathbf{f}^{(17)}\left(q_{1}, q_{3}\right), 3.18 \leq q_{3} \leq 3.54 \text { and } 0 \leq q_{1} \leq 240^{\circ}
\end{aligned}
$$




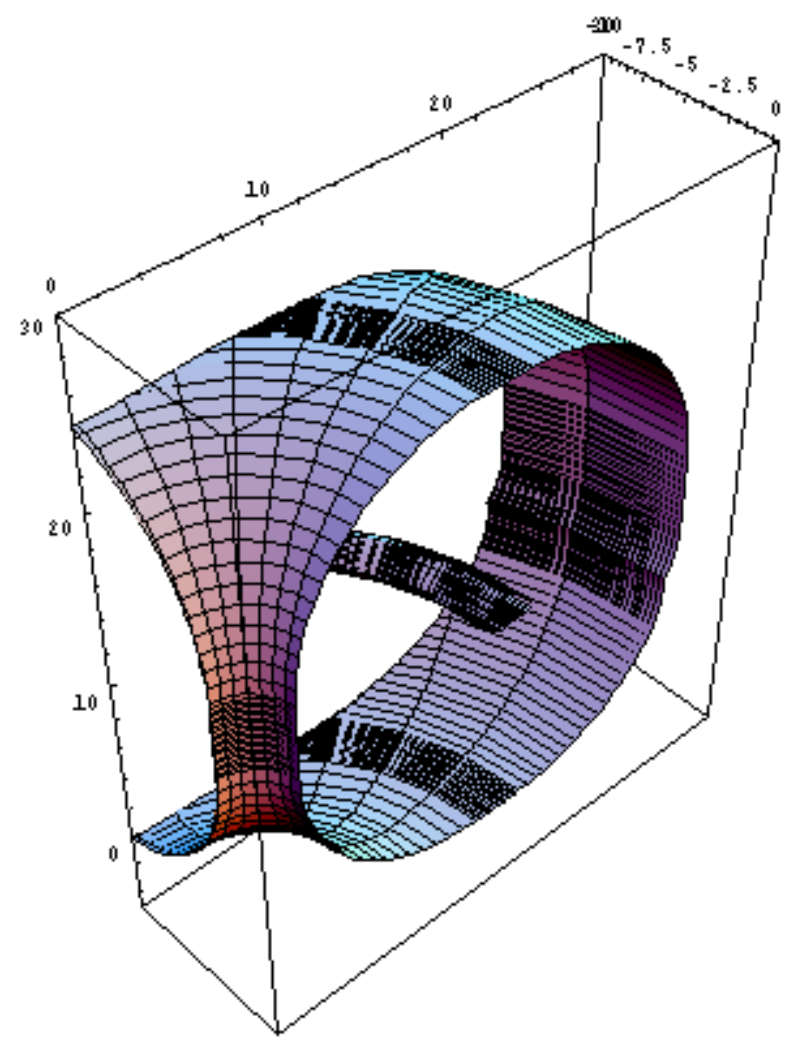

Figure 15 A cross section of the workspace depicting the boundary and void surface patches

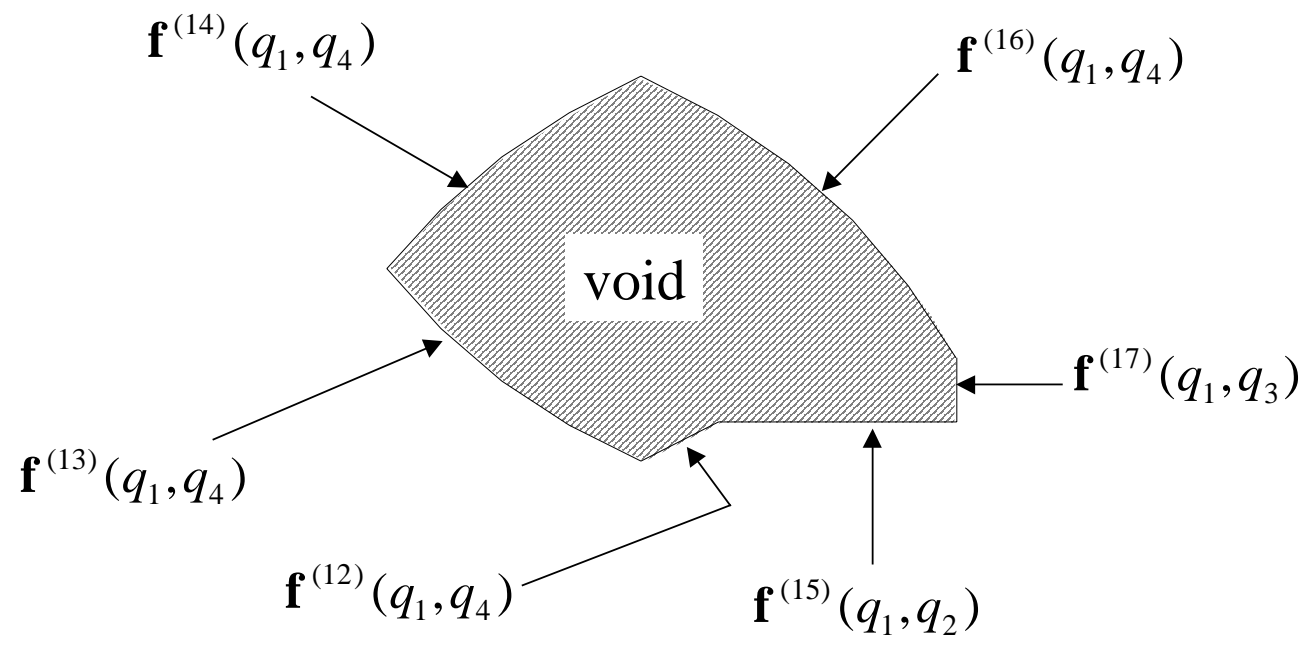

Figure 16 Boundary surfaces (traces of surface patches) to a void

\section{CONCLUSIONS}

A broadly applicable formulation for the determination of voids in the workspace of serial mechanical manipulators has been introduced. Based on acceleration analyses of an endeffector point on a singular surface, the difference in normal acceleration components yields a quadratic form. 
It was shown that definiteness properties of the quadratic form are used to delineate surface regions that admit motion output normal in the direction of the normal vector at this point. It is emphasized that this work is possible only because of the parametric nature of the singular surfaces delineated using the rank deficiency criteria introduced elsewhere.

While some surface patches in the workspace admit no motion in the normal direction, only enclosures that are bound by such surface patches are identified as a boundary. It was shown that those surface patches that do not admit motion but are not part of the boundary are indeed impediments to motion and may cause the manipulator to exhibit control difficulties. It was also shown that the interior boundary (boundary to voids) and the exterior boundary of the workspace can be determined using this method. The presented formulation is capable of delineating the external boundary of a general n-DOF manipulator.

Compared with the work by Cecarelli and Vinciquerra (1995), holes and voids are equally treated since the differentiation between an apple and a ring void has no significance using the proposed mathematical formulation. In the work by the two authors, a hole in the workspace is identified as a region of unreachable points containing at least a straight line that passes through it without making contact with the workspace.

The formulation presented herein and its accompanying experimental code demonstrate an effective method for understanding the kinematics inside the workspace of serial manipulators.

\section{REFERENCES}

Abdel-Malek, K. and Yeh, H. J., 2000 "Local Dexterity Analysis for Open Kinematic Chains," Mechanism and Machine Theory, Vol. 35, pp. 131-154.

Abdel-Malek, K. and Yeh, H.J., 1997a "Analytical Boundary of the Workspace for General Three Degree-of-Freedom Mechanisms," International Journal of Robotics Research. Vol. 16, No. 2, pp. 198-213.

Abdel-Malek, K. and Yeh, H.J., 1997b, "Geometric Representation of the Swept Volume Using Jacobian Rank-Deficiency Conditions," Computer Aided Design, Vol. 29, No. 6, pp. 457-468.

Abdel-Malek, K., 1997, "Determination of the Accessible Output Set of a General Three

Degree of Freedom Manipulator Arm" Robotics and Computer-Integrated

Manufacturing, Vol. 13, No. 1, pp. 63-72

Abdel-Malek, K., Adkins, F., Yeh, H.J., and Haug, E.J., 1997, "On the Determination of Boundaries to Manipulator Workspaces," Robotics and Computer-Integrated Manufacturing, Vol. 13, No. 1, pp.63-72.

Abdel-Malek, K., Yeh, H-J, and Khairallah, N., 1999a, "Workspace, Void, and Volume

Determination of the General 5DOF Manipulator, Mechanics of Structures and

Machines, 27(1), 91-117.

Abdel-Malek, Yeh, H., and Alkam, N., 1999b, "Control barriers of Positioning

Manipulators", Proceedings of the 25th Design Automation in Mechanisms and Robotics, Las Vegas, Nevada. 
Agrawal, S.K. 1990, "Workspace Boundaries of In-parallel Manipulator Systems," Int. J. of Robotics and Automation, Vol. 7, No. 2, pp. 94-99.

Ahmad, S. and Luo, S. 1988, "Analysis of kinematic singularities for robot manipulators in Cartesian coordinate parameters", Proceedings of the IEEE Int. Conf. on Rob. and Autom., Philadelphia, PA, 840-845.

Allgower, E. L. and Georg, K. 1990. Numerical Continuation Methods: An Introduction. Springer-Verlag, Berlin.

Ceccarelli, M. and Vinciguerra, A., 1995, "On the Workspace of General 4R Manipulators," International Journal of Robotics Research, Vol. 14, No. 2, pp. 152-160.

Ceccarelli, M., 1995, "A Synthesis Algorithm for Three-Revolute Manipulators by Using an Algebraic Formulation of Workspace Boundary," ASME J. of Mechanical Design, Vol. 117, No. 2(A), pp. 298-302.

Chevallereau, C. 1996. Feasible trajectories for a non redundant robot at a singularity. Proc. of IEEE Int. Conf. on Rob. and Aut., Minneapolis, MN, pp. 1871-1876.

Davidson, J.K., and Hunt, K.H., 1987, "Rigid Body Location and Robot Workspace: Some Alternative Manipulator Forms," ASME J. of Mechanisms, Transmissions, and Automation in Design, 109(2), 224-232.

Denavit, J. and Hartenberg, R.S., 1955. A kinematic notation for lower-pair mechanisms based on matrices. Journal of Applied Mechanics, 77:215-221.

Emiris, D.M., 1993, "Workspace Analysis of Realistic Elbow and Dual-elbow Robot," Mechanisms and Machine Theory, Vol. 28, No. 3, pp. 375-396.

Farin, G., 1993, Curves and Surfaces for Computer-Aided Geometric Design, Academic Press, London, UK.

Fu, K. S. Gonzalez, R. C. and Lee, C. S. G. 1987. Robotics: Control, Sensing, Vision, and Intelligence, McGraw-Hill, Inc., New York.

Gorla, B. 1981. Influence of the control on the structure of a manipulator from a kinematic point of view. Proc. 4th Symp. Thoery and Practice of Rob. Manipulators, Zaborow, Poland, 30-46.

Gosselin, C. and Angeles, J., 1990, "Singularity Analysis of Closed Loop Kinematic Chains," IEEE Trans. on Robotics and Automation, Vol. 6, No. 3, pp. 281-290.

Gupta, K.G. and Roth, B. 1982, "Design Considerations for Manipulator Workspace," ASME Journal of Mechanical Design, Vol. 104, No. 4, pp. 704-711.

Haug, E. J. Adkins, F. A. Qiu, C. C. and Yen, J. 1995. Analysis of barriers to control of manipulators within accessible output sets. Proceedings of the 20th ASME Design Engineering Technical Conference, Boston, MA, 82:697-704.

Haug, E.J., Luh, C.M., Adkins, F.A., and Wang, 1996, "Numerical algorithms for mapping boundaries of manipulator workspaces,” Trans. ASME J. Mech. Des., 118 (1), 228-234

Haug, E.J., Luh, C.M., Adkins, F.A., and Wang, J.Y., 1996, "Numerical algorithms for mapping boundaries of manipulator workspaces," ASME Journal of Mechanical Design, 118, pp. 228-234.

Kumar, A. and Waldron, K.J., 1981, “The Workspace of a Mechanical Manipulator," ASME J. Mech. Design, Vol. 103, pp. 665-672.

Kumar, V., 1985, Robot Manipulators-Workspaces and Geometric Dexterity, Masters Thesis, The Ohio State University.

Lai, Z. C. and Yang, D. C. H. 1984. On the singularity analysis of simple six-link manipulators ASME Paper No. 84-DET-220. 
Lai, Z. C. and Yang, D. C. H. 1986. A new method for the singularity analysis of simple sixlink manipulators. Int. J. of Rob. Res., 5(2):66-74.

Lipkin, H. and Pohl, E. 1991. Enumeration of singular configurations for robotic manipulators. ASME J. Mech., Trans., and Autom. in Des., 113:272-279.

Litvin, F. L. Fanghella, P. Tan, J. and Zhang, Y. 1986a. Singularities in motion and dispplacement functions of spppatial linkages. ASME J. of Mech., Trans., and Autom. in Des., 108(4):516-523.

Litvin, F. L. Yi, Z. Castelli, V. P. and Innocenti, C. 1986b. Singularities, configurations, and displacement functions for manipulators. Int. J. Rob. Res., 5(2):52-65.

Merlet, J. P. 1989. Singular configurations of parallel manipulators and Grassman geometry. Int. J. of Rob. Res., 8:45-56.

Mohammadi Daniali, H. R., Zsombor-Murray, P. J. and Angeles, J. 1995. Singularity analysis of planar parallel manipulators. Mechanism and Machine Theory, 30:665-678.

Nielsen, L. deWit, C. C. and Hagander, P. 1991. Controllability issues of robots in singular configurations. Proceedings of IEEE Int. Conf. on Rob. and Autom., Sacramento, CA.

Oblak, D. and Kohli, D., 1988. Boundary surfaces, limit surfaces, crossable and noncrossable surfaces in workspace of mechanical manipulators. ASME Journal of Mechanisms, Transmissions, and Automation in Design, (110):389-396.

Pai, D. K. and Leu, M. C. 1992. Generecity and singularities of robot manipulators. IEEE Trans. on Rob. Autom., 8:545-559.

Pennock, G.R. and Kassner, D.J., 1993, "The Workspace of a General Planar Three-Degreeof-Freedom Platform-Type Manipulator," ASME Journal of Mechanical Design, Vol. 115, pp. 269-276.

Shamir, T. 1990. The singularities of redundant robot arms. Int. J. of Rob. Res., 2(1):113121.

Shu, M. Kohli, D. and Dwivedi, S. H. 1986. Proceedings of the 6th World Congress on Theory of Machines and Mechanisms, New Delhi, India, 988-993.

Soylu, R. and Duffy, J. 1988. Hypersurfaces of special configurations of serial manipulators and related concepts. Part II: Passive joints, configurations, component manifolds and some applications. J. Rob. Systems, 5:31-53.

Spanos, J. and Kohli, D. 1985 "Workspace Analysis of Regional Sttructure of Manipulators," ASME J. of Mech. Trans. and Aut. in Design, Vol. 107, pp. 219-225.

Sugimoto, K. Duffy, J. andHunt, K. H. 1982. Special configurations of spatial mechanisms and robot arms. Mechanism and Machine Theory, 117(2):119-132.

Tourassis, V. D. and Ang, M. H. 1992. Identification and analysis of robot manipulator singularities. Int. J. of Rob. Res., 11:248-259.

Tsai, Y.C. and Soni, A.H., 1981, "Accessible Region and Synthesis of Robot Arm," ASME Journal of Mechanical Design, Vol. 103, pp. 803-811.

Waldron, K. J. 1987. Operating barriers within the workspace of manipulators. Proceedings of the Society of Manufacturing Engineers, Chicago, IL, Robots II/17th ISIR, 8:35-46.

Waldron, K. J. Wang, S. L. and Bolin, S. L. 1985. A study of the Jacobian matrix of serial manipulators. ASME J. of Mech., Trans., and Autom. in Des., 107(2):230-238.

Wang, J.Y. and Wu, J.K., 1993, "Dexterous Workspaces of Manipulators, Part 2: Computational Methods," Mechanics of Structures and Machines, Vol. 21, No. 4, pp. 471-506. 
Wang, S. L. and Waldron, K. J. 1987. Study of the singular configurations of serial manipulators. ASME J. of Mech., Trans., and Autom. in Des., 109(1):14-20.

Yeh, H.J. 1996, Singularity and Workspace Analyses of Serial Robot Manipulators. PhD Thesis, The University of Iowa, Iowa City, IA, December 1996.

Zhang, S.J., Sanger, D.J., and Howard, D., 1996, "Workspaces of a Walking Machine and their Graphical Representation. Part I: kinematic workspaces," Robotica, 14, pp. 71-79 